\title{
Genetic diversity of Giardia isolates from patients in Chandigarh region: India
}

\author{
Shabnam Thakur, Upninder Kaur and Rakesh Sehgal * (D)
}

\begin{abstract}
Objective: The aim of study was to characterize Giardia isolates genetically among patients in Chandigarh region, India. For this, nested PCR targeting fragment of the glutamate dehydrogenase (GLUD1 earlier named as GDH) gene was used. Phylogenetic analysis was done by constructing neighbor-joining tree made out of the nucleotide sequences of $G$. intestinalis isolates obtained in this study and with the known sequences published in GenBank.

Results: Out of 40 samples, GLUD1 gene was amplified in 33 samples (82.5\%). The product of GLUD1 gene was successfully sequenced only in 32 samples. In these samples, assemblage B was found in 27 (84.37\%) samples whereas 5 (15.6\%) samples had assemblage A. Among assemblage B most of them were of BIII. Therefore, genotyping of Giardia would be helpful in conducting epidemiological studies.
\end{abstract}

Keywords: Giardia, Genotype, GLUD1 (glutamate dehydrogenase), PCR, Sequencing

\section{Introduction}

Giardia intestinalis is well known intestinal parasite of humans and mammals. Giardia causes approximately 280 million cases of giardiasis worldwide annually [1]. Most of these cases are associated with lower socioeconomic status. In the year 2004, giardiasis was included in WHO 'Neglected Diseases Initiative' because of its high prevalence in communities with low socio-economic status.

Giardiasis is acquired due to ingestion of cysts of Giardia in water or food [2]. Giardia intestinalis is composed of eight major genotypes or assemblages $(\mathrm{A}-\mathrm{H})$ [2]. Genotype A and B are common among humans having variable distribution frequency in different geographical locations and these assemblages mainly considered as zoonotic assemblages as they are able to infect both men and animals [3]. These assemblages are further divided into subassemblages on the basis of either digestion by restriction enzyme or sequence analysis. Assemblages A are classified as AI, AII,AIII and AIV. Subassemblages AI

*Correspondence: sehgalpgi@gmail.com

Department of Medical Parasitology, Postgraduate Institute of Medical

Research and Education, Chandigarh 160012, India and AII were commonly found in humans while AI, AIII and AIV are subassemblages of animals. Zoonotic potential is linked with only subassemblage AI [4]. Assemblage B, catogorised into four sub-assemblages BI, BII, BIII and BIV. As per literature subassemblages BIII and BIV were reported in humans while other two are specific for animals $[4,5]$. The BIII sub-assemblage is closer to subassemblages BI and BII and therefore has zoonotic potential. The GLUD1 (earlier known as GDH) locus has been utilized for genetic characterization of $G$. intestinalis isolates in vertebrates [4] hosts and is able to categorize them into sub genotypes/subassemblages. The present work was aimed to determine assemblages and subassemblages of Giardia isolates involved in its transmission by using glutamate dehydrogenase (GLUD1) marker.

\section{Main text \\ Materials and methods \\ Sample collection}

Forty microscopic Giardia positive stool samples were collected from the Routine Laboratory of Department of Medical Parasitology, PGIMER, Chandigarh from August 2019 to December 2019. 
Table 1 PCR results of samples with their assemblages

\begin{tabular}{|c|c|c|}
\hline Sample No & PCR & Assemblages \\
\hline 1 & +ve & $B$ \\
\hline 2 & +ve & B \\
\hline 3 & +ve & $B$ \\
\hline 4 & +ve & $B$ \\
\hline 5 & +ve & $B$ \\
\hline 6 & +ve & $B$ \\
\hline 7 & +ve & $B$ \\
\hline 8 & +ve & $\mathrm{B}$ \\
\hline 9 & +ve & $B$ \\
\hline 10 & +ve & $\mathrm{B}$ \\
\hline 11 & +ve & $B$ \\
\hline 12 & +ve & $\mathrm{B}$ \\
\hline 13 & +ve & Unable to sequence \\
\hline 14 & +ve & $B$ \\
\hline 15 & +ve & B \\
\hline 16 & +ve & $B$ \\
\hline 17 & +ve & B \\
\hline 18 & +ve & $\mathrm{B}$ \\
\hline 19 & +ve & $B$ \\
\hline 20 & +ve & $\mathrm{B}$ \\
\hline 21 & +ve & $A$ \\
\hline 22 & +ve & $B$ \\
\hline 23 & -ve & - \\
\hline 24 & -ve & - \\
\hline 25 & -ve & - \\
\hline 26 & -ve & - \\
\hline 27 & -ve & - \\
\hline 28 & +ve & $B$ \\
\hline 29 & +ve & $B$ \\
\hline 30 & +ve & $A$ \\
\hline 31 & +ve & $B$ \\
\hline 32 & -ve & - \\
\hline 33 & +ve & $A$ \\
\hline 34 & +ve & $B$ \\
\hline 35 & -ve & - \\
\hline 36 & +ve & $B$ \\
\hline 37 & +ve & $B$ \\
\hline 38 & +ve & $A$ \\
\hline 39 & +ve & $A$ \\
\hline 40 & +ve & B \\
\hline
\end{tabular}

\section{DNA extraction}

From stool samples, DNA was extracted by using QIAmp Fast DNA Stool Mini Kit (QIAGEN, Germany) as per manufacturer's instructions with slight modifications. The suspension was initially incubated at $90^{\circ} \mathrm{C}$ for $15 \mathrm{~min}$ and then for another $30 \mathrm{~min}$ at $75{ }^{\circ} \mathrm{C}$. DNA was eluted in
$50 \mu \mathrm{l}$ of $\mathrm{AE}$ buffer. DNA concentration was measured by NanoQuant (Infinite ${ }^{\circledR} 200$ PRO NanoQuant) and stored at $-20^{\circ} \mathrm{C}$ until further use.

\section{Polymerase chain reaction amplification}

The two-step PCR was employed for the amplification of GLUD1 gene (432 bp) by using previously published primers given by Read et al. [6]. The conditions and primers for both primary and secondary reactions are given as Additional file 1: Table S1. The first set of PCR reaction comprised of $2.0 \mu \mathrm{L}$ of DNA template, $12.5 \mu \mathrm{L} 2 \times$ Go Taq Green Mix, $1 \mu \mathrm{L}$ of each primer $(10 \mu \mathrm{M}), 1 \mu \mathrm{L}$ of Bovine Serum Albumin (BSA) whereas in case of secondary PCR, DNA template was replaced by the product of primary reaction. For the negative control, nuclease-free water and for the positive control, DNA of Giardia strain, Portland 1 was used for each PCR reaction. All the precautions were taken to prevent contamination.

\section{$P C R$ product purification and sequencing}

All PCR-positive samples were sequenced using secondary primers. PCR products were sequenced in both forward and reverse directions. By using BLAST, nucleotide similarity of sequenced amplicons was searched in GenBank (http://www.ncbi.nlm.nih.gov/blast). CLUSTAL X was used to determine multiple sequence alignments. Neighbor-joining distance trees were prepared using MEGAX software (https://www.megasoftwa re.net/) (Fig. 1). Bootstrap values were based on 1000 replicas. All the sequences obtained during the study were submitted to the GenBank (Accession number: MT584168-MT584199).

The direct links which are publicly available are as follows:

https://www.ncbi.nlm.nih.gov/nuccore/MT584168 https://www.ncbi.nlm.nih.gov/nuccore/MT584169 https://www.ncbi.nlm.nih.gov/nuccore/MT584170 https://www.ncbi.nlm.nih.gov/nuccore/MT584171 https://www.ncbi.nlm.nih.gov/nuccore/MT584172 https://www.ncbi.nlm.nih.gov/nuccore/MT584173 https://www.ncbi.nlm.nih.gov/nuccore/MT584174 https://www.ncbi.nlm.nih.gov/nuccore/MT584175 https://www.ncbi.nlm.nih.gov/nuccore/MT584176 https://www.ncbi.nlm.nih.gov/nuccore/MT584177 https://www.ncbi.nlm.nih.gov/nuccore/MT584178 https://www.ncbi.nlm.nih.gov/nuccore/MT584179 https://www.ncbi.nlm.nih.gov/nuccore/MT584180 https://www.ncbi.nlm.nih.gov/nuccore/MT584181 https://www.ncbi.nlm.nih.gov/nuccore/MT584182 https://www.ncbi.nlm.nih.gov/nuccore/MT584183 https://www.ncbi.nlm.nih.gov/nuccore/MT584184 https://www.ncbi.nlm.nih.gov/nuccore/MT584185 
https://www.ncbi.nlm.nih.gov/nuccore/MT584186

https://www.ncbi.nlm.nih.gov/nuccore/MT584187

https://www.ncbi.nlm.nih.gov/nuccore/MT584188

https://www.ncbi.nlm.nih.gov/nuccore/MT584189

https://www.ncbi.nlm.nih.gov/nuccore/MT584190

https://www.ncbi.nlm.nih.gov/nuccore/MT584191

https://www.ncbi.nlm.nih.gov/nuccore/MT584192

https://www.ncbi.nlm.nih.gov/nuccore/MT584193

https://www.ncbi.nlm.nih.gov/nuccore/MT584194

https://www.ncbi.nlm.nih.gov/nuccore/MT584195

https://www.ncbi.nlm.nih.gov/nuccore/MT584196

https://www.ncbi.nlm.nih.gov/nuccore/MT584197

https://www.ncbi.nlm.nih.gov/nuccore/MT584198

https://www.ncbi.nlm.nih.gov/nuccore/MT584199

\section{Results}

Out of 40 samples, the GLUD1 gene was amplified in 33 samples (82.5\%) (Table1, Fig. 2). The possible reason for this occurrence indicated the less parasitic load in these isolated samples. The product of GLUD1 gene was successfully sequenced only in 32 samples. In these samples, assemblage B was found in 27 (84.37\%) among which 18 (66.66\%) were sub-genotype BIII and 9 (33.33\%) were sub-genotype BIV samples whereas 5 (15.6\%) samples had assemblage A. Four of them were AI subgenotype and only1 belonged to sub-genotype AII.

\section{Discussion}

Giardia intestinalis is the most common and frequent intestinal parasitic agent of gastroenteritis mainly in the developing countries [7]. The present study provides data on genetic diversity of Giardia isolates from patients in the Chandigarh region. As per results, assemblage $\mathrm{A}$ and $\mathrm{B}$ are common among this population, which is in concordance to other studies [8, 9]. In our study, assemblage $\mathrm{B}$ was the predominant genotype observed followed by assemblage A. However, similar observations were previously reported and observed worldwide which showed that assemblage $\mathrm{B}$ was predominant in comparison to assemblage $A$ [9-12]. There are studies which showed the presence of assemblage B in Rhesus macaques (Macaca mulatta) and potable water resources of Northern India $[13,14]$. But other studies have reported the assemblage A as the predominant genotype in other regions [15]. Due to geographical variations, differences were observed in the prevalence of various genotypes and the detection of these variations would be helpful in designing effective therapeutic approaches.

\section{Conclusion}

The results showed that PCR sequencing and phylogenetic analysis is an excellent molecular technique for genotyping of Giardia intestinalis. Detection of Giardia intestinalis assemblages and sub-assemblageswould be helpful in conducting epidemiological studies.

\section{Limitation of study}

Present study involves only single locus for genotyping and also the sample size is less so it is difficult to interpret zoonotic potential of these isolates. Therefore, multi-locus typing data is required to differentiate between Giardia isolates. 


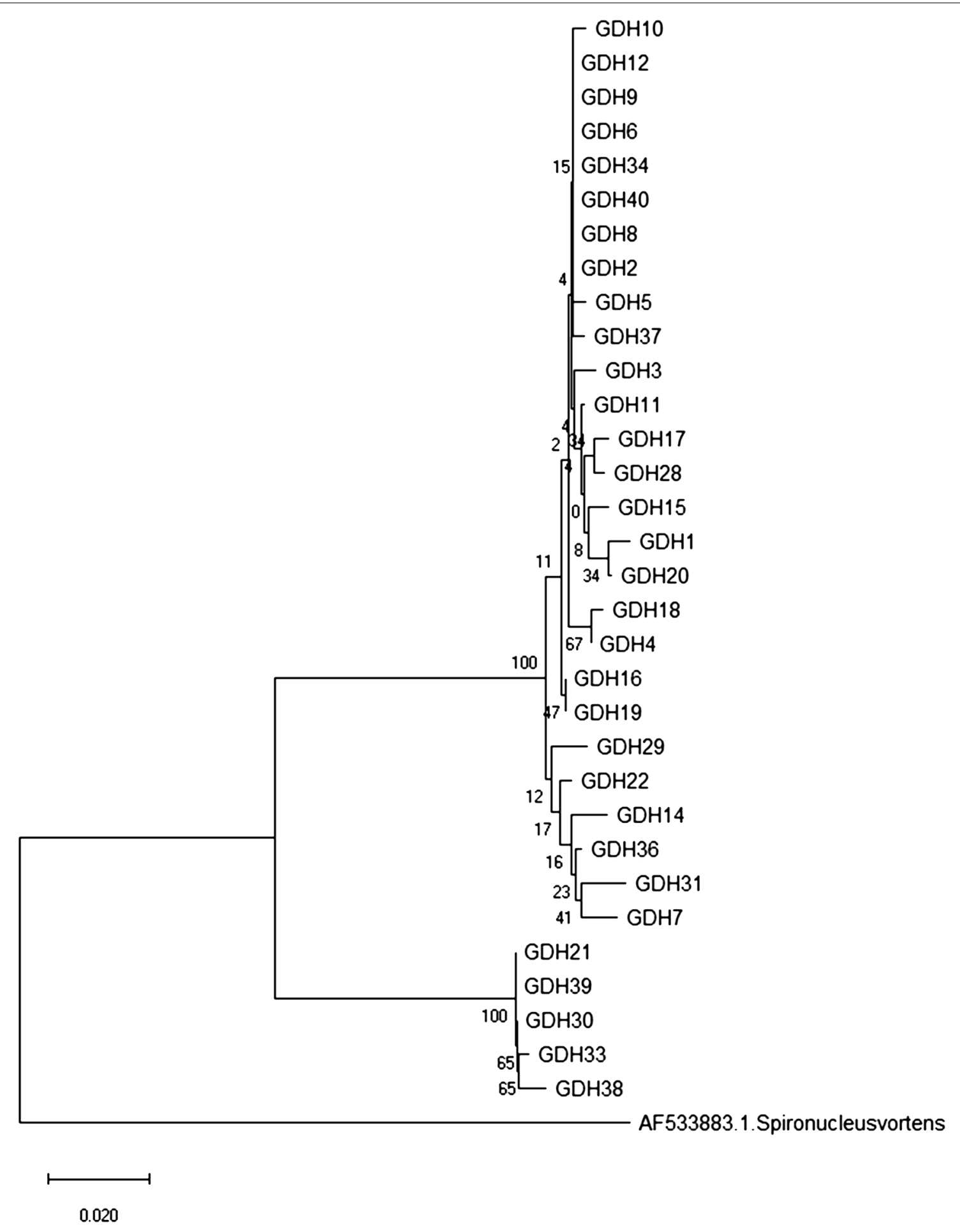

Fig. 1 Phylogenetic tree constructed with the neighbor-joining method using nucleotide sequences of GLUD1 gene. The sequence of Spironucleus vortens was used as an out-group 


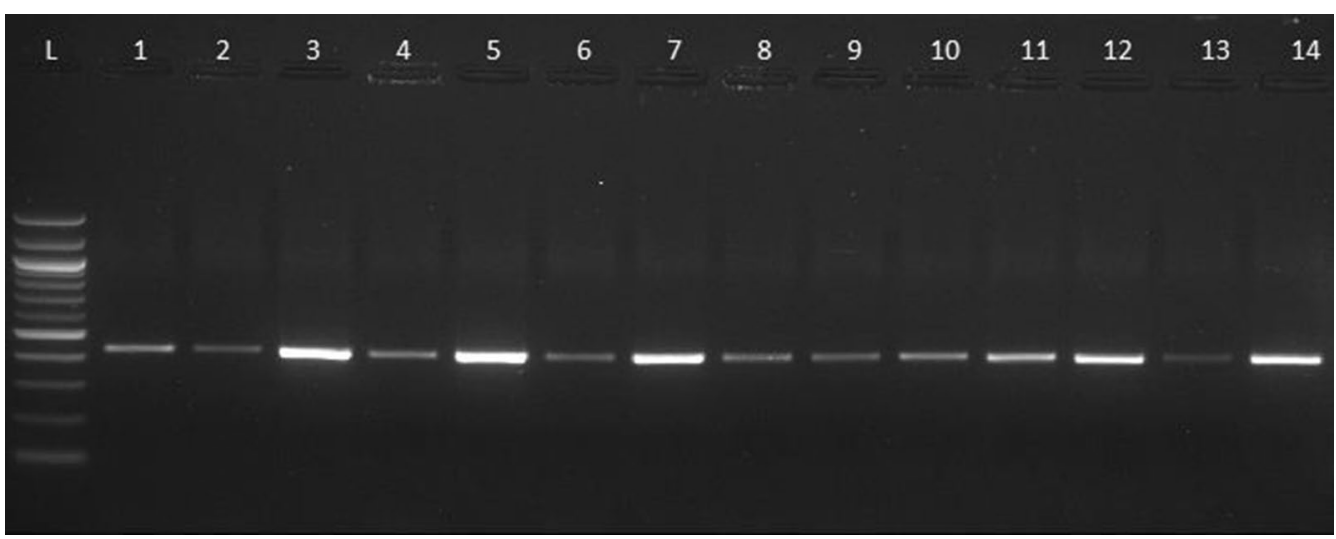

Fig. 2 Nested-PCR amplification of Giardia intestinalis GLUD1 gene (432bp) 1.5\% agarose gel stained with Ethidium bromide. L, ladder (100bp); lane1-13, Giardia positive samples; lane14, positive control

\section{Supplementary Information}

The online version contains supplementary material available at https://doi. org/10.1186/s13104-020-05419-1.

Additional file 1: Table S1. Sequence of primers and sgRNA were listed.

\section{Abbreviations}

BLAST: Basic Local Alignment Search Tool; DNA: Deoxyribonucleic Acid; PCR: Polymerase Chain Reaction; BSA: Bovine Serum Albumin.

\section{Acknowledgements}

Authors duly acknowledge Ms Sofia Behad, Senior Research Fellow for providing assistance in alignment of sequences

\section{Authors' contributions}

ST planned \& conducted all the practical work in the laboratory. UK helped in preparing \& checking the manuscript. RS helped in designing and drafting the work, provided financial assistance for completing the present work and also helped in analysis of the data of the present work. All authors read and approved the final manuscript.

\section{Funding}

None.

\section{Availability of data and materials}

All the supporting data related to the present work is available with the authors and the sequences obtained during the study were submitted to the GenBank (Accession number: MT584168-MT584199).

\section{Ethics approval and consent to participate}

The study has been approved by Institutional Ethics Committee, PGIMER Chandigarh vide IEC No- 04/2017-601. Patients were informed of the study objectives and voluntary written consent was sought and obtained before inclusion.

\section{Consent for publication}

All the authors have agreed to publish the present research paper.

\section{Competing interests}

There are no financial, general and institutional competing interests.

Received: 20 Auqust 2020 Accepted: 11 December 2020

Published online: 19 January 2021

\section{References}

1. Einarsson E, Maayeh S, Svärd SG. An up-date on Giardia and giardiasis. Curr Opin Microbiol. 2016;34:47-52.

2. Feng $Y$, Xiao L. Zoonotic potential and molecular epidemiology of Giardia species and giardiasis. Clin Microbiol Rev. 2011;24(1):110-40.

3. Durigan M, Abreu AG, Zucchi MI, Franco RM, de Souza AP. Genetic diversity of Giardia duodenalis: multilocus genotyping reveals zoonotic potential between clinical and environmental sources in a metropolitan region of Brazil. PLoS ONE. 2014;9(12):e115489.

4. Hooshyar H, Ghafarinasab S, Arbabi M, Delavari M, Rasti S. Genetic variation of Giardia lamblia isolates from food-handlers in Kashan, Central Iran. Iran J Parasitol. 2017;12(1):83.

5. Heyworth MF. Giardia duodenalis genetic assemblages and hosts. Parasite. 2016;23:13.

6. Read C, Monis P, Thompson R. Discrimination of all genotypes of Giardia duodenalis at the glutamate dehydrogenase locus using PCR-RFLP. Infect Genet Evol. 2004;4(2):125-30.

7. Thompson RC, Smith A. Zoonotic enteric protozoa. Vet Parasitol. 2011:182:70-8.

8. Ajjampur SS, Sankaran P, Kannan A, Sathyakumar K, Sarkar R, Gladstone BP, Kang G. Giardia duodenalis assemblages associated with diarrhea in children in South India identified by PCR-RFLP. Am J Trop Med Hyg. 2009;80(1):16-9.

9. Laishram S, Kannan A, Rajendran P, Kang G, Ajjampur SSR. Mixed Giardia duodenalis assemblage infections in children and adults in South India. Epidemiol Infect. 2012;140:2023-7.

10. Minvielle MC, Molina NB, Polverino D, Basualdo JA. First genotyping of Giardia lamblia from human and animal feces in Argentina. S Am Mem Inst Oswaldo Cruz. 2008;103:98-103.

11. Tak V, Mirdha BR, Yadav P, Vyas P, Makharia GK, Bhatnagar S. Molecular characterisation of Giardia intestinalis assemblages from human isolates at a tertiary care centre of India. Indian J Med Microbiol. 2014;32(1):19.

12. Chanu NO, Singh TS, Dutta S. Detection and genetic characterization of Giardia intestinalis in children with gastrointestinal symptoms by PCR RFLP in Sikkim, India. J Nat Sci Biol Med. 2018;9(2):193.

13. Debenham JJ, Tysnes K, Khunger S, Robertson LJ. Occurrence of Giardia, Cryptosporidium, and Entamoeba in wild rhesus macaques (Macaca mulatta) living in urban and semi-rural North-West India. Int J Parasitol Parasites Wildl. 2017;6(1):29-34.

14. Utaaker KS, Joshi H, Kumar A, Chaudhary S, Robertson LJ. Occurrence of Cryptosporidium and Giardia in potable water sources in Chandigarh, Northern India. J Water Supply Res Technol AQUA. 2019;68(6):483-94.

15. Skhal D, Aboualchamat G, AI Mariri A, AI NS. Prevalence of Giardia duodenalis assemblages and sub-assemblages in symptomatic patients from Damascus city and its suburbs. Infect Genet Evol. 2017;47:155-60.

\section{Publisher's Note}

Springer Nature remains neutral with regard to jurisdictional claims in published maps and institutional affiliations. 